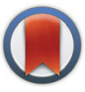

CrossMark $\leftarrow$ click for updates

Cite this: Polym. Chem., 2016, 7, 6293

Received 30th August 2016,

Accepted 30th September 2016

DOI: 10.1039/c6py01523a

www.rsc.org/polymers

\section{Glyconanoparticles with controlled morphologies and their interactions with a dendritic cell lectin $\uparrow$}

\author{
Gokhan Yilmaz, a,b,c Lea Messager, ${ }^{d}$ Anne S. Gleinich, ${ }^{e}$ Daniel A. Mitchell, \\ Giuseppe Battaglia*d and C. Remzi Becer*c
}

\begin{abstract}
Well-defined amphiphilic block glycopolymers with equal mannose content have been self-assembled in aqueous solution to form glyconanoparticles with different morphologies. The size and shape of nanoparticles have significant effects on the interactions with the dendritic cell-specific intercellular adhesion molecule-3grabbing non-integrin (DC-SIGN; CD209), characterized using a surface plasmon resonance spectrometer (SPR).
\end{abstract}

Protein-carbohydrate interactions play an important role in many biological processes including cell interactions with immune systems, tumor metastasis, adhesion of infectious agents to host cells and many more. ${ }^{1}$ Proteins involved in these signaling and processing pathways are known as lectins. ${ }^{2}$ These carbohydrate binding proteins are wide-spread in nature and they differ from antibodies or enzymes entities. ${ }^{3}$ For example, it has been shown that some viruses such as HIV express many carbohydrate entities at their surface, which enable them to bind to these lectins on the cell surface in the immune system. Therefore, one of the promising strategies for fighting against infectious diseases would be to design competing systems with higher lectin affinity than pathogens, thus preventing their adhesion.

Recent and elegant synthetic routes have allowed polymer chemists to prepare a wide range of glyco-polymers/particles that provide strong and selective recognition properties towards lectins. ${ }^{4-8}$ In particular, amphiphilic block glycopolymers (GPs) have attracted a great attention in terms of their ability to form various types of glyconanoparticles. These

\footnotetext{
${ }^{a}$ Department of Chemistry, University of Warwick, CV4 7AL Coventry, UK

${ }^{b}$ Department of Basic Sciences, Turkish Military Academy, Ankara, Turkey ${ }^{c}$ Polymer Chemistry Laboratory, School of Engineering and Materials Science, Queen Mary, University of London, E1 4NS London, UK. E-mail: r.becer@qmul.ac.uk ${ }^{d}$ Department of Chemistry, University College London, WC1H OAJ London, UK ${ }^{e}$ Clinical Sciences Research Institute, Warwick Medical School,

University of Warwick, CV2 2DX Coventry, UK

$\dagger$ Electronic supplementary information (ESI) available: Synthesis and characterization of the polymers. See DOI: 10.1039/c6py01523a
}

amphiphilic GPs are generally composed of biocompatible, biodegradable hydrophobic polymer blocks covalently bonded to a biocompatible hydrophilic block. ${ }^{9-11}$ Some recent studies have shown that amphiphilic block glycopolymers with different carbohydrate compositions were used to produce nanoparticles, such as micelles, nanospheres, core-shell nanoparticles, micelle-like nanoparticles, crew cut micelles, nanocapsules and polymersomes. ${ }^{12-16}$ These studies facilitate the understanding and investigation of GP-lectin binding activities that are significantly influenced by GP architecture, valency, size, and density of binding elements. Furthermore, they offer a promising route for the creation of a broad variety of bioactive self-assembled glyco-nanostructures for biomedical applications such as drug delivery, biomaterials, bioand nanotechnologies, and gene therapy. ${ }^{17-24}$ In the last few years, the number of strategies devoted to prepare GPs via single electron transfer living radical polymerization (SET-LRP) polymerization technique has increased rapidly. Therefore, SET-LRP was used in this study to synthesize several types of amphiphilic block glycocopolymers bearing mannose moieties by using methyl acrylate (MA) and a glycomonomer (ManAc) and/or poly(ethylene glycol) (PEG) as a hydrophobic and a hydrophilic block, respectively. Mannose glycomonomer was prepared according to the procedure reported by Zhang $e t a{ }^{25}$ Poly(ethylene glycol) 2-bromoisobutyrate (PEG-Br) initiator was used as an initiator to prepare amphiphilic triblock glycocopolymer. The self-assembly behaviors of these well-defined amphiphilic GPs were investigated in aqueous solution to obtain glyconanostructures with various sizes and morphologies. The resulting glyconanoparticles (GNPs) were further investigated for their binding affinity towards lectins, in particular with DC-SIGN, which is a C-type human lectin present on both macrophages and also dendritic cell subpopulations. DC-SIGN binds to microorganisms and host molecules by recognizing surfaces rich in mannose containing glycans through multivalent glycanprotein interactions and notably serves as a target molecule for several viruses such as HIV and hepatitis C virus. ${ }^{26}$ 
Here, we report the synthesis of well-defined amphiphilic GPs using the SET-LRP technique, their spontaneous selfassembly in aqueous solution, and binding studies of the resulting glyconanoparticles with DC-SIGN.

A series of well-defined amphiphilic block GPs were prepared via SET-LRP using EBiB or PEG-Br initiator. The molar ratio of $[\mathrm{MA}] /[\mathrm{ManAc}]$ has been varied to yield amphiphilic block copolymers with increasing ratios between their hydrophobic and hydrophilic moieties. One-pot polymerization approach has been employed to ensure well-defined block copolymer formation. Initially, MA was polymerized up to high conversion and subsequently; the chain extension with mannose glycomonomer ( $\mathrm{DP}=15$, dissolved and degassed in $1 \mathrm{~mL}$ of DMSO) to form the second block was carried out (ESI, Fig. S7 $\dagger$ ). The monomer conversions were followed by ${ }^{1} \mathrm{H}$ NMR, which showed the disappearance of vinyl groups apparent between 5.8-6.4 ppm. The purified glycopolymers were further analyzed by GPC and ${ }^{1} \mathrm{H}$ NMR (ESI, Fig. S9†). Based on these results, it is evident that the copolymerization of MA and ManAc has been achieved with a good control as indicated by low polydispersity indices and increasing molecular weight of the block copolymer. Analysis of all $\left.\mathrm{P}\left((\mathrm{MA})_{m}-b \text {-(ManAc }\right)_{n}\right)$ polymers (P1, P2, P3, and P4) are presented in Table 1. High chain end fidelity of $\mathrm{P}((\mathrm{PEG})-b$-(MA)) has been confirmed by chain extension with MA prior to the synthesis of triblock glycocopolymer, P5, using the same approach in the presence of PEG-Br initiator. GPC analysis (ESI, Fig. S10 $\dagger$ ) revealed a clear baseline shift of elution traces after each monomer addition with increasing molecular weight and final PDI of 1.17. No significant tailing or shoulders have been observed in the GPC throughout the polymerization. Moreover, the measured $M_{\mathrm{n}},{ }_{\mathrm{GPC}}$ and the $M_{\mathrm{n} \text {,theo }}$ calculated from the monomer conversions are in good agreement.

Nanoparticles of these amphiphilic GPs having PEG block and/or carbohydrate bearing chains as hydrophilic block and MA as hydrophobic one were prepared using a nanoprecipitation method an the detailed conditions are summarized in the ESI. $\dagger$ Slow-injection of water droplets into glycopolymer in DMF solution ensures to obtain thermodynamically stable selfassembled structures. The morphology and size of the resulting nanoparticles were systematically analyzed by TEM and DLS.
Amphiphilic block copolymers P1, P2 and P3 yield welldispersed nanoparticles of around $25 \mathrm{~nm}$. While P1 and P2 generated micelles with a regular spherical shape, P3 created non-uniform micelles due to their non-regular spherical shape according to TEM imaging and DLS measurements. These features are typical of spherical micelles. Further TEM analysis was conducted on each suspension measuring the average diameter of more than 50 single particles. The majority of the particles display an average diameter of $20 \mathrm{~nm}$, which is slightly smaller than the values obtained by DLS, thus providing the dry structure of these particles. Furthermore, TEM imaging shows that most of the particles are not clustered, which is promising, as micelles generally tend to cluster together. Moreover, it was also observed that P2 and P3 yielded small amount of larger aggregates, which could potentially be vesicles. Increasing the hydrophobic content of the copolymer revealed the formation of self-assembled nanoparticles with larger hydrodynamic diameter. Indeed, the TEM image of P4 indicated the formation of vesicular structures and DLS measurements showed a narrow size distribution centered at $380 \mathrm{~nm}$.

According to TEM and DLS results, sizes of glyconanoparticles are increasing with increasing of polymer length (ESI, Table S2 $\dagger$ ). Depending on the increasing MA fraction in the polymer, the existence region of nanoparticles has become broader. This is possibly due to dependency on composition, molecular geometry, relative block lengths of the constitutive copolymers, and the preparation methods on the formation of glyconanoparticles. Another attempt was conducted on P4 to obtain a higher number of vesicular particles, decreasing the injection rate of the aqueous solution.

The self-assembly of amphiphilic triblock GP, P5, has lower hydrophilic fraction than P1-P4, $\mathrm{P}\left((\mathrm{MA})_{m}-b-(\mathrm{ManAc})_{n}\right)$, due to the increasing molecular weight ratio of the hydrophobic block. According to TEM measurement, the majority of nanoparticles were worm-like micelles but with a small fraction of spherical micelles (Fig. 1). The length of these worm-like micelles is in the range of 45 to $60 \mathrm{~nm}$ and their width is $24 \mathrm{~nm}$, which is in agreement with unimer chain scaling. Further increase of molar ratio of hydrophobic block (for P4 and P5) allowed fabricating different aggregates such as vesicles and worm-like micelles.

Table 1 Summary of monomer conversions, number average molecular weight $\left(M_{n}\right)$ and molecular weight distributions ( ()$)$ of SET-LRP polymerization of all amphiphilic block glycocopolymers

\begin{tabular}{|c|c|c|c|c|c|c|c|}
\hline \multirow[b]{2}{*}{ Run } & \multirow[b]{2}{*}{ Polymer } & \multirow[b]{2}{*}[\mathrm{MA}]{$:[\mathrm{ManAc}]:[\mathrm{I}]^{a}$} & \multicolumn{2}{|c|}{ Conv. $^{b}(\%)$} & \multirow[b]{2}{*}{$M_{\mathrm{n}, \mathrm{NMR}}{ }^{c}\left(\mathrm{~g} \mathrm{~mol}^{-1}\right)$} & \multirow[b]{2}{*}{$M_{\mathrm{n}, \mathrm{GPC}}{ }^{d}\left(\mathrm{~g} \mathrm{~mol}^{-1}\right)$} & \multirow[b]{2}{*}{$Ð$} \\
\hline & & & MA & ManAc & & & \\
\hline $\mathbf{P 2}$ & $\mathrm{P}\left((\mathrm{MA})_{103}-b-(\mathrm{ManAc})_{15}\right)$ & $105: 15: 1$ & 97 & 99 & 14150 & 14800 & 1.18 \\
\hline P3 & $\mathrm{P}\left((\mathrm{MA})_{130}-b-(\mathrm{ManAc})_{15}\right)$ & $135: 15: 1$ & 96 & 99 & 16400 & 17600 & 1.17 \\
\hline $\mathbf{P 4}$ & $\mathrm{P}\left((\mathrm{MA})_{206^{-}}-(\mathrm{ManAc})_{15}\right)$ & $210: 15: 1$ & 97 & 98 & 22800 & 26900 & 1.20 \\
\hline P5 & $\mathrm{P}\left((\mathrm{PEG})_{45}-b-(\mathrm{MA})_{172}-b-(\mathrm{ManAc})_{15}\right)$ & $180: 15: 1$ & 96 & 99 & 23800 & 24600 & 1.17 \\
\hline
\end{tabular}

${ }^{a}$ Initial molar ratio of monomers to initiator. ${ }^{b}$ Conversions obtained from ${ }^{1} \mathrm{H}$ NMR analysis. ${ }^{c}$ Calculated according to equation $M_{\mathrm{n}, \mathrm{NMR}}=\left([\mathrm{M}]_{\mathrm{o}} /\right.$ $[\mathrm{I}] \times$ NMR conv. $\% \times$ MW of MA and ManAc) + MW of initiator. ${ }^{d}$ Determined by GPC (DMF as an eluent and relative to PMMA standards). 


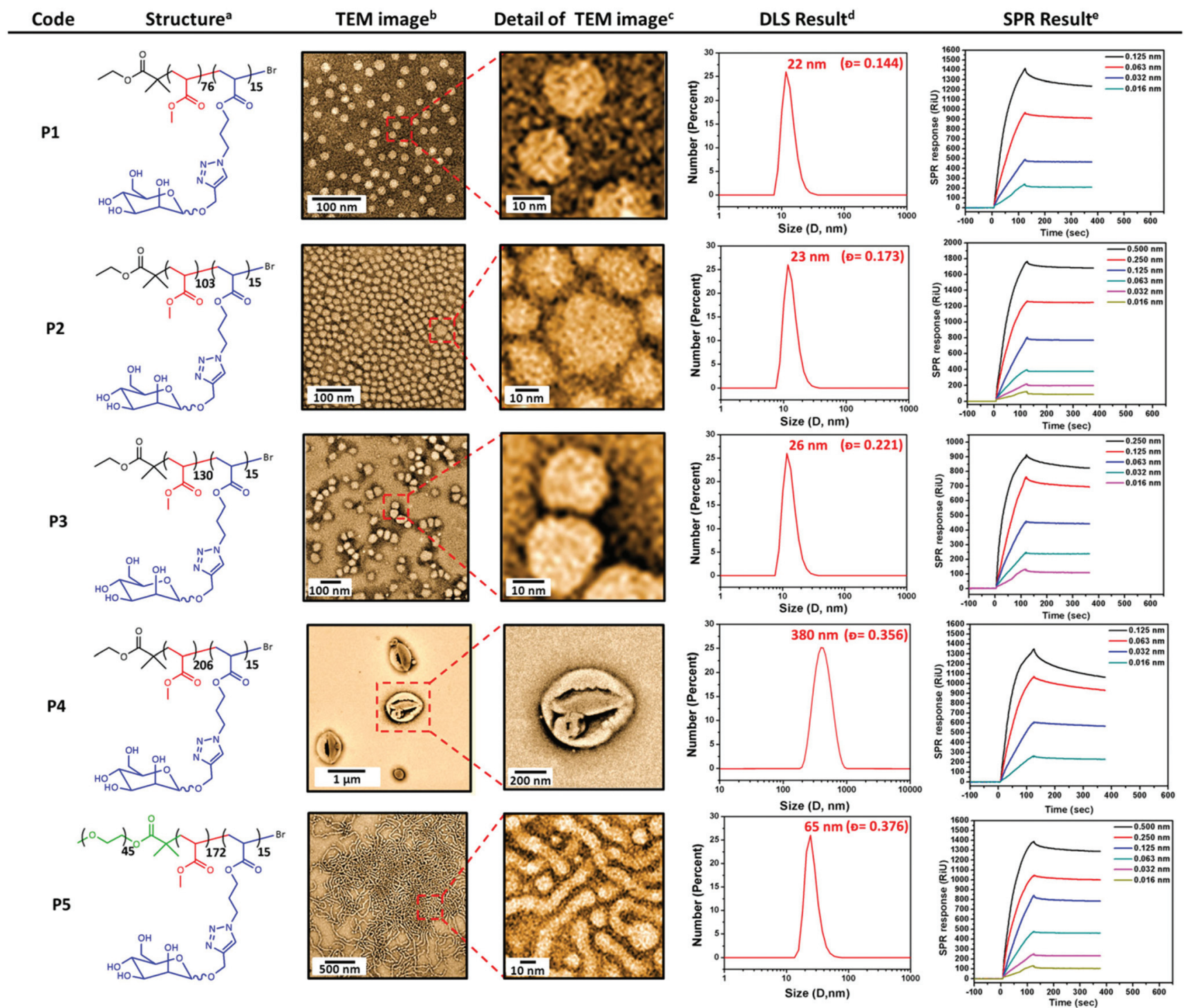

Fig. 1 Characterization of glyconanoparticles and their respective binding with DC-SIGN. [a] Chemical structures of polymers; [b] TEM images of glyconanoparticles in selected solvent condition; [c] zoomed in TEM images; [d] size of glyconanoparticles via DLS [e] DC-SIGN binding of glyconanoparticles measured by SPR.

The biological activity of these nanoparticles towards lectins was measured via SPR. DC-SIGN, which is a human lectin on cell surfaces that preferentially binds to $\mathrm{N}$-linked high-mannose oligosaccharides, was employed for binding assays. DC-SIGN plays a critical role in human immunodeficiency virus (HIV) trafficking ability to interact with the highly mannosylated gp120 glycoprotein present on the envelope of HIV. In general, all glyconanoparticles showed strong binding signal with DC-SIGN (Fig. 1).

As expected, the binding of nanoparticles to DC-SIGN decreased at lower nanoparticle concentrations. All glyconanoparticles exhibited relatively high binding values at higher concentrations. Therefore, in order to compare the binding levels of these glyconanoparticles, the nanoparticle concentration of all nanoparticle solutions were kept constant to determine the effect of particle size on DC-SIGN binding (Fig. 2). According to the SPR data, vesicle structured nanoparticles $\mathbf{P 4}$ had a much higher affinity in comparison to other GNPs. Surprisingly, P1 showed the second highest binding level in this series after P4. This may be due to the mannose groups on the surface of $\mathbf{P 1}$ glyconanoparticles being more accessible in order to mediate strong interactions with DC-SIGN. Another interesting result is that the worm-like micelles P5 showed higher level of interaction than $\mathbf{P} 2$ and P3. The possible explanation for this can be that the formation of worm-like micelles in clustered structures may enhance the binding capability of nanoparticles with the natural oligomeric structure of DC-SIGN. As a result, the small increase in the size of a nano- 


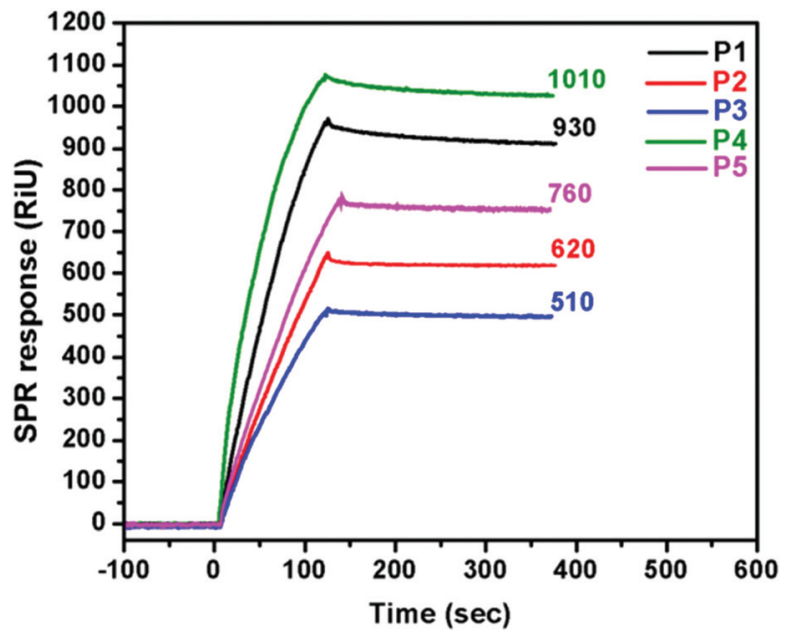

Fig. 2 SPR sensorgrams showing the binding of glyconanoparticles with DC-SIGN at the same nanoparticle concentration (0.063 nM).

particle showed a significant decrease on binding with DC-SIGN.

\section{Conclusions}

In summary, several types of amphiphilic block co-glycopolymers with optimal molecular weights and relatively narrow molecular weight distributions were synthesized via SET-LRP to prepare different glycopolymer nanostructures. These synthesized amphiphilic glycopolymers with the same number of mannose units self-assembled in water to generate glyconanoparticles with different morphologies such as spherical and worm-like micelles as well as spherical vesicles. The shape and size of these self-assembled glyconanoparticles were characterized via TEM and DLS. Finally, the interaction of these glyconanoparticles of different size and shape with DC-SIGN was monitored by SPR. The SPR results indicate that the size and shape of nanoparticles have significant effect on the binding level with DC-SIGN. According to the binding performance of these glycopolymer nanostructures, they can be potentially utilized in biomedical applications such as cell-targeted drug delivery and inhibition of viral infection such as HIV.

\section{Acknowledgements}

This work is supported by Turkish Armed Forces, and by the European Commission (EU-ITN EuroSequences Proposal No: 642083). A. S. G is supported by a studentship from The General Charity of the City of Coventry.

\section{References}

1 M. Ambrosi, N. R. Cameron and B. G. Davis, Biomol. Chem., 2005, 3, 1593.
2 C. R. Bertozzi and L. L. Kiessling, Science, 2001, 291, 2357.

3 J. Arnaud, A. Audfray and A. Imberty, Chem. Soc. Rev., 2013, 42, 4798.

4 L. Wang, G. R. Williams, H. I. Nie, J. Quan and L. M. Zhu, Polym. Chem., 2014, 5, 3009.

5 Q. Zhang, L. Su, J. Collins, R. Wallis, D. Mitchell, D. M. Haddleton and C. R. Becer, J. Am. Chem. Soc., 2014, 2, 4325 .

6 G. Yilmaz and C. R. Becer, Polym. Chem., 2015, 6, 5503.

7 W. Wang, D. Chance, V. Mossine and T. Mawhinney, Glycoconjugate J., 2014, 31, 133.

8 G. Yilmaz and C. R. Becer, Eur. Polym. J., 2013, 49, 3046.

9 A. Muñoz-Bonilla, O. León, V. Bordegé, M. Sánchez-Chaves and M. Fernández-García, J. Polym. Sci., Part A: Polym. Chem., 2013, 51, 1337.

10 M. Alvárez-Paino, R. Juan-Rodríguez, R. Cuervo-Rodríguez, A. Muñoz-Bonilla and M. Fernández-García, J. Colloid Interface Sci., 2014, 417, 336.

11 F. Suriano, R. Pratt, J. P. K. Tan, N. Wiradharma, A. Nelson, Y. Y. Yang, P. Dubois and J. L. Hedrick, Biomaterials, 2010, 31, 2637.

12 A. Peyret, J. F. Trant, C. V. Bonduelle, K. Ferji, N. Jain, S. Lecommandoux and E. R. Gillies, Polym. Chem., 2015, 6, 7902.

13 S. Vandewalle, S. Wallyn, S. Chattopadhyay, C. R. Becer and F. Du Prez, Eur. Polym. J., 2015, 69, 490.

14 I. Kurtulus, G. Yilmaz, M. Ucuncu, M. Emrullahoglu, C. R. Becer and V. Bulmus, Polym. Chem., 2014, 5, 1593.

15 K. Godula and C. R. Bertozzi, J. Am. Chem. Soc., 2012, 134, 15732.

16 S. F. Lou, L. Wang, G. R. Williams, H. Nie, J. Quan and L. Zhu, Colloids Surf., B, 2014, 113, 368.

17 K. Babiuch, R. Wyrwa, K. Wagner, T. Seemann, S. Hoeppener, C. R. Becer, R. Linke, M. Gottschaldt, J. R. Weisser, M. Schnabelrauch and U. S. Schubert, Biomacromolecules, 2011, 12, 681.

18 Y. Luo, L. Liu, X. Wang, H. Shi, W. Lv and J. Li, J. Soft Matter, 2012, 8, 1634.

19 C. Y. Zhang, H. C. Yeh, M. T. Kuroki and T. H. Wang, Nat. Mater., 2005, 4, 826.

20 T. G. Iversen, T. Skotland and K. Sandvig, Nano Today, 2011, 6, 176.

21 D. W. Lim, Y. I. Yeom and T. G. Park, Bioconjugate Chem., 2000, 11, 688.

22 E. Mahon, T. Aastrup and T. Barboiu, Chem. Commun., 2010, 46, 5491.

23 E. Mahon, T. Aastrup and T. Barboiu, Chem. Commun., 2010, 46, 2441.

24 A. Dag, J. Zhao and M. H. Stenzel, ACS Macro Lett., 2015, 4, 579.

25 Q. Zhang, J. Collins, A. Anastasaki, R. Wallis, D. A. Mitchell, C. R. Becer and D. M. Haddleton, Angew. Chem., Int. Ed., 2013, 52, 4435.

26 T. B. H. Geijtenbeek, D. S. Kwon, R. Torensma, S. J. van Vliet, G. C. F. van Duijnhoven, J. Middel, I. L. M. H. A. Cornelissen, H. S. L. M. Nottet, V. N. KewalRamani, D. R. Littman, C. G. Figdor and Y. van Kooyk, Cell, 2000, 100, 587. 